Article

\title{
Online Parameter Identification of Lithium-Ion Batteries Using a Novel Multiple Forgetting Factor Recursive Least Square Algorithm
}

\author{
Bizhong Xia ${ }^{1}$, Rui Huang ${ }^{1, *}$, Zizhou Lao ${ }^{1}$, Ruifeng Zhang ${ }^{1,2}{ }^{\oplus}$, Yongzhi Lai ${ }^{2}$, Weiwei Zheng ${ }^{2}$, \\ Huawen Wang ${ }^{2}$, Wei Wang ${ }^{2}$ and Mingwang Wang ${ }^{2}$ \\ 1 Graduate School at Shenzhen, Tsinghua University, Shenzhen 518055, China; \\ xiabz@sz.tsinghua.edu.cn (B.X.); zzlao@foxmail.com (Z.L.); zhang.ruifeng@sz.tsinghua.edu.cn (R.Z.) \\ 2 Sunwoda Electronic Co. Ltd., Shenzhen 518108, China; lyz@sunwoda.com (Y.L.); \\ zhww@sunwoda.com (W.Z.); wanghuawen@sunwoda.com (H.W.); willian-wang@sunwoda.com (W.W.); \\ yetongzhou@sunwoda.com (M.W.) \\ * Correspondence: huangr17@mails.tsinghua.edu.cn; Tel.: +86-131-2016-5530
}

Received: 19 October 2018; Accepted: 12 November 2018; Published: 16 November 2018

\begin{abstract}
The model parameters of the lithium-ion battery are of great importance to model-based battery state estimation methods. The fact that parameters change in different rates with operation temperature, state of charge (SOC), state of health $(\mathrm{SOH})$ and other factors calls for an online parameter identification algorithm that can track different dynamic characters of the parameters. In this paper, a novel multiple forgetting factor recursive least square (MFFRLS) algorithm was proposed. Forgetting factors were assigned to each parameter, allowing the algorithm to capture the different dynamics of the parameters. Particle swarm optimization (PSO) was utilized to determine the optimal forgetting factors. A state of the art SOC estimator, known as the unscented Kalman filter (UKF), was combined with the online parameter identification to create an accurate estimation of SOC. The effectiveness of the proposed method was verified through a driving cycle under constant temperature and three different driving cycles under varied temperature. The single forgetting factor recursive least square (SFFRLS)-UKF and UKF with fixed parameter were also tested for comparison. The proposed MFFRLS-UKF method obtained an accurate estimation of SOC especially when the battery was running in an environment of changing temperature.
\end{abstract}

Keywords: battery management system; state of charge estimation; multiple forgetting factor; recursive least square; online parameter identification

\section{Introduction}

Environmental crisis and fossil fuel depletion call for the implementation of new energy vehicles (NEVs) in recent years [1]. The electric vehicle (EV) is a promising candidate among all kinds of NEVs [2]. Lithium-ion batteries have been widely used in EVs for its advantages of high energy density, high power density, long cycle life, no memory effect, and low self-discharging rate [3]. However, safety concerns arise as the lithium-ion batteries must be controlled to operate within specific ranges of temperature and voltage [4], the thermal behavior regarding different load conditions should be considered [5]. In addition, the state of the battery should be estimated, such as state of charge (SOC), state of health ( $\mathrm{SOH}$ ) [6], etc. The battery management system (BMS) is required to provide precise controls and an accurate state estimation of batteries.

Among those functions of BMS, SOC estimation is of great importance. An accurate SOC estimation is critical for power distribution strategy and responsible for the prevention of over-charging 
and over-discharging [7]. However, SOC represents the ratio of remaining electricity to the nominal capacity and cannot be measured directly. Researchers have been focusing on the improvement of the accuracy of SOC estimation results by using measurable variables, including operation current, terminal voltage, etc.

To date, numerous methods for SOC estimation have been proposed to achieve this purpose, many of which are model-based methods. Compared with the non-model based counterparts such as the coulomb counting and open circuit voltage (OCV)-SOC look-up table method [8,9], model-based methods have the merit of overcoming input disturbances due to the close-loop correction process. In addition, the accuracy and fast convergence of model-based estimation can be realized when combined with state of the art observers or filters based on modern control theory. The Kalman filter family is widely utilized to estimate the SOC, such as the extended Kalman filter (EKF) [10,11], unscented Kalman filter (UKF) [12,13], or the cubature Kalman filter (CKF) [14,15], and they successfully deal with the high nonlinearity of the battery. A particle filter is also investigated and applied in SOC estimation [16]. Observer based methods have been employed for battery models, such as the sliding mode observer [2,17], nonlinear observer [18], and H-infinity observer [19,20]. These model-based algorithms mentioned above use fixed parameters identified offline by specific experiments. However, it should be noted that the values of the model parameters are not constant, instead, they are affected by various factors depending on the cell chemistry, including the ambient temperature, the current and voltage profiles, and the aging of the battery. For example, the aging state plays a fundamental role in the parameters related to the hysteresis effect in lithium iron phosphate batteries [21], while the model parameters of nickel manganese cobalt oxide batteries are greatly influenced by temperatures [22]. The discrepancies between offline data and actual value of model parameters may cause significant impact on the accuracy of model output. Therefore, an algorithm that can update model parameters online according to the measurable variables is preferable to improve the performance of SOC estimation.

Considering the above-mentioned circumstances, several approaches have been proposed to counteract the effect of parameter variation. In [23], the online estimation of model parameters is realized by $\mathrm{H}$-infinity filter. Tang et al. [24] used a model migration method to compensate model uncertainties caused by SOC, SOH, and temperature. He et al. [25] proposed a SOC and internal resistance joint estimation algorithm based on UKF. Xiong et al. [26] used EKF to update battery parameters. Based on the minimization of the sum of squared errors, the least squares (LS) algorithm and its derivatives are easy to be applied to the auto regressive exogenous (ARX) model [27], many of which are recursive versions, such as the recursive least square (RLS) [28]. In [29], a recursive total least square (RTLS) algorithm is utilized to address the problem caused by unexpected noises. Wei et al. [30] proposed a Frisch scheme based bias compensating RLS to attenuate the noise caused bias. A moving-window LS scheme has been implemented in [31]. In Ref. [32], a recursive extended least square (RELS) method, which includes a moving average noise, is applied to capture the battery dynamics. In [33], the identification of slow dynamics and fast dynamics are executed separately using the EKF and RLS respectively. The fast tracking ability of RLS can be improved by introducing a forgetting factor which controls the influence of past data on the recent estimation. The SFFRLS have been investigated in [34,35], where the impacts of different parameters vanish in the same rate. However, the parameters of the battery have different dynamic characters. To address this conflicting problem, MFFRLS algorithms have been introduced. In [36], the MFFRLS scheme is realized by solving the governing function directly. In Refs. $[37,38]$, the adaptive MFFRLS algorithm has been implemented whose MFF scheme is developed by Vahidi et al. in Ref. [39], in which the covariance error of each parameter is updated separately. Uosaki el al. [40] proposed a MFFRLS method in which the parameter estimates are formulated as the weighted sum of the estimates obtained by multiple SFFRLS methods operating in parallel with different forgetting factors. A method to realize MFF is based on U-D factorization introduced by Hardier [41]. In Ref. [42], a vector-type forgetting factor is employed to keep different parameters on track. 
In this paper, a novel MFF strategy is proposed and applied to improve the performance of RLS. A first-order RC model is established and the parameters of which are identified by a novel MFFRLS method whose forgetting factors are optimized by PSO. UKF is utilized to estimate the battery SOC and combined with the online parameter identification scheme. A series of experiments conducted under different temperature variations indicates that the proposed MFFRLS-UKF algorithm estimates the SOC accurately. Compared with SFFRLS-UKF and UKF, it exhibits greater capability to track the battery dynamics in situations where the ambient temperature of the battery changes significantly.

The rest of this paper is organized as follows: Section 2 introduces the details of battery modeling and procedures of UKF estimator used for SOC prediction. In Section 3, the novel MFFRLS algorithm is presented and applied to the battery model. The optimization of forgetting factors using the PSO method is introduced. The joint algorithm of MFFRLS and UKF is shown. Section 4 presents the experimental configuration and verification of the proposed method. Conclusions are drawn in Section 5.

\section{Battery Modeling and State of Charge Estimation Scheme}

\subsection{Model Selection of the Battery}

It is a fundamental task to define the type of model in a model-based SOC estimation method. A suitable model should be selected to obtain an accurate state estimation. Numerous models have been devised to describe the characters of the battery, most of which can be classified into two groups: the electrochemical model and the equivalent circuit model (ECM). The electrochemical models, based on the theories of electrochemical reactions, provides a more accurate simulation of battery terminal voltage as well as internal states [43]. However, the complex partial differential equations and large number of parameters needed make it difficult to be embedded in a real BMS. The ECMs use circuit components to simulate the electric characters of the battery. The commonly used ECMs include the first-order resistor-capacitor (1-RC) model, also known as the Thevenin Model, in which the capacitor simulates the transient voltage behavior with respect to time [44]; the second-order resistor-capacitor (2-RC) model, known as the partnership for a new generation of vehicles (PNGV) Model; and other higher-order ECM models. Typically, the higher the order of the ECM, the more accurate result it can yield since there are more components that can simulate the dynamic and complex behavior of the battery, but the complexity increases simultaneously. In [45], 12 different ECMs were studied and comparison results suggest that the first order RC model is preferred for its capability to strike a balance between model complexity and model accuracy. Therefore, the 1-RC model is chosen, as shown in Figure 1. The resistor $R_{0}$ stands for ohmic resistance, which is influenced by circle life and ambient temperature. The parallel $R_{p}$ and $C_{p}$ simulate the polarization effect of the battery. The open-circuit voltage $U_{o c}$ is a monotonic function of SOC and the relationship can be determined by experiments introduced in Section 4.2. $U_{p}$ and $U_{t}$ represent the polarization voltage and the terminal voltage respectively, and $i$ is the load current whose positive value stands for discharging and negative value denotes charging.

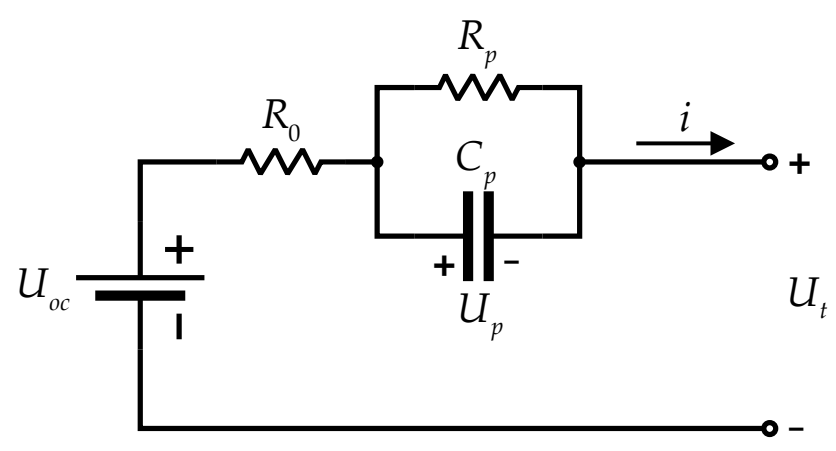

Figure 1. Schematic diagram of a first-order RC model. 
The electrical characteristic of the model can be described as the equations below according to Kirchhoff's law.

$$
\begin{gathered}
C_{p} \frac{d U_{p}}{d t}+\frac{U_{p}}{R_{p}}=i \\
U_{o c}=U_{t}+i R_{0}+U_{p}
\end{gathered}
$$

\subsection{Nonlinear Kalman Filter Based SOC Estimation}

In this paper, UKF is utilized to estimate the SOC for its capability to deal with system nonlinearity. SOC and polarization voltage $U_{p}$ are chosen as the state variable in the state-space model. The input and output of the model are load current $i$ and terminal voltage $U_{t}$, respectively. Using the coulomb counting method, the derivative of SOC can be expressed in Equation (3):

$$
\mathrm{S} \dot{\mathrm{OC}}=-\frac{1}{\mathrm{C}_{n}} i
$$

where $C_{n}$ is the nominal capacity. The discrete version of the model can be established for UKF adoption in Equations (4) and (5), in which $T_{S}$ is the sampling time.

$$
\begin{gathered}
\left(\begin{array}{l}
\operatorname{SOC}(k) \\
U_{p}(k)
\end{array}\right)=\left(\begin{array}{cc}
1 & 0 \\
0 & 1-\frac{T_{s}}{C_{p} R_{p}}
\end{array}\right)\left(\begin{array}{l}
\operatorname{SOC}(k-1) \\
U_{p}(k-1)
\end{array}\right)+\left(\begin{array}{c}
-\frac{T_{s}}{C_{n}} \\
\frac{T_{s}}{C_{p}}
\end{array}\right) i(k) \\
U_{t}(k)=U_{o c}(k)-i(k) R_{0}-U_{p}(k)
\end{gathered}
$$

The SOC estimation based on UKF is summarized in Table 1.

Table 1. Major steps of state of charge (SOC) estimation based on the unscented Kalman filter (UKF).

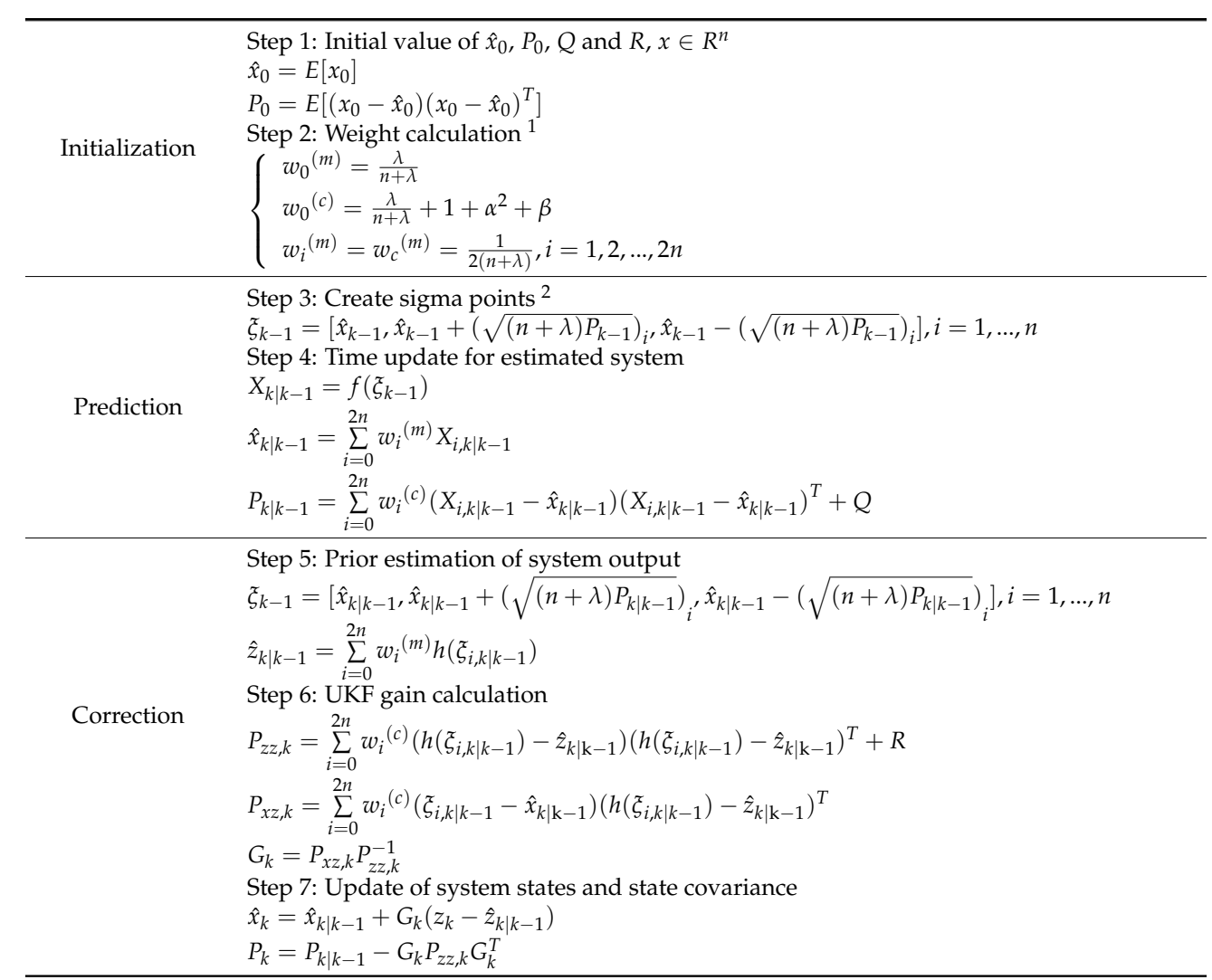

${ }^{1} \alpha$ is a constant between 0 and $1, \beta$ is set to 2 here for the Gaussian distribution hypothesis, and $\lambda$ is a scalar parameter determined by $\lambda=\alpha^{2}(n+\kappa)-n$, where $\kappa$ is usually set to zero. ${ }^{2}\left(\sqrt{(n+\lambda) P_{k-1}}\right)_{i}$ represents the $i$-th column vector of the square root of $(n+\lambda) P_{k-1}$. 


\section{A Novel Multiple Forgetting Factor Recursive Least Square Method}

The parameters of the ECM of the battery vary with numerous factors such as operation temperature, $\mathrm{SOC}, \mathrm{SOH}$ and so on, resulting in an unreliable state estimation result if constant values are adopted, especially when the ambient environment of the battery is changing. The online identification method updates the model parameters constantly and is preferable for a high precision state estimation. The recursive least square method gains its popularity for its capability to capture the variation of the parameters in a single input single output (SISO) system, meanwhile less storage is needed and fast convergence can be realized.

\subsection{Single Forgetting Factoc Recursive Least Square Algorithm}

To reduce the influence of the old samples and focus more on recent data, the forgetting factor is employed in the RLS. The commonly used single forgetting factor RLS scheme is described as follows.

Consider the SISO linear discrete time-varying system described in Equation (6):

$$
y(k)=\varphi^{T}(k) \theta+\zeta(k)
$$

in which

$$
\left\{\begin{array}{l}
\varphi(k)=\left[-y(k-1), \ldots,-y\left(k-n_{a}\right), u(k-d), \ldots, u\left(k-d-n_{b}\right)\right]^{T} \\
\theta=\left[a_{1}, \ldots, a_{n a}, b_{0}, \ldots, b_{n b}\right]^{T}
\end{array}\right.
$$

$\zeta(k)$ is additive white noise, $n_{a}, n_{b}$ and $d$ are system constants. The forgetting factor $\lambda$ is introduced to exponentially decrease the remote data in the least square error function $V$ shown in Equation (8), in which $L$ is the number of observations:

$$
V=\sum_{k=1}^{L} \lambda^{L-k}\left[y(k)-\varphi^{T}(k) \hat{\theta}\right]^{2}
$$

The minimum value of the least square error function $V$ can be obtained by calculating the estimated $\hat{\theta}$ recursively using the following Equation (9).

$$
\left\{\begin{array}{l}
\hat{\theta}(k)=\hat{\theta}(k-1)+K(k)\left[y(k)-\varphi^{T}(k) \hat{\theta}(k-1)\right] \\
K(k)=\frac{P(k-1) \varphi(k)}{\lambda+\varphi^{T}(k) P(k-1) \varphi(k)} \\
P(k)=\frac{1}{\lambda}\left[I-K(k) \varphi^{T}(k)\right] P(k-1)
\end{array}\right.
$$

The smaller the value of $\lambda$, the stronger the ability of tracking since the old record die out rapidly. However, fast tracking may cause instability of the estimation result. Usually the range of the forgetting factor is set to $[0.9,1]$ to strike a balance in this trade-off relationship.

\subsection{Introducing the Multiple Forgetting Factors Scheme}

In Section 3.1, the SFFRLS was introduced, the use of a forgetting factor makes the estimation keep track of the change of new samples. However, there is only one forgetting factor that applies to all the parameters to be estimated. In fact, the parameters usually vary at different rates. For example, the internal resistance $R_{0}$ of the battery varies with temperature, SOC and $\mathrm{SOH}$ while the polarization resistance $R_{p}$ is influenced by the highly dynamic load current [37]. As a consequence, employing a forgetting factor to parameters which have different dynamic features will lead to the underperformance of the algorithm. The discrepancies of dynamic characteristics of parameters require different and independent forgetting factors for parameters. The RLS with multiple forgetting factors was recommended to meet the requirement. The forgetting factors can be assigned independently so that the fast changing parameters have smaller forgetting factors while the steadily varying parameters have larger forgetting factors. Inspired by the MFFRLS introduced by 
Francesco et al. in [46], we propose a novel MFFRLS scheme in this paper for the online identification of battery parameters as:

$$
\left\{\begin{array}{l}
\hat{\theta}(k)=\hat{\theta}(k-1)+K(k)\left[y(k)-\varphi^{T}(k) \hat{\theta}(k-1)\right] \\
K(k)=\frac{F_{\lambda}\left(P^{-1}(k-1)\right)^{-1} \varphi(k)}{1+\varphi^{T}(k) F_{\lambda}\left(P^{-1}(k-1)\right)^{-1} \varphi(k)} \\
P(k)=\left[I-K(k) \varphi^{T}(k)\right] F_{\lambda}\left(P^{-1}(k-1)\right)^{-1}
\end{array}\right.
$$

in which $F_{\lambda}$ is a forgetting map defined as follows:

$$
\left[F_{\lambda}(M)\right]_{i, j}=\left\{\begin{array}{lr}
\min \left(\lambda_{1}, \ldots, \lambda_{n}\right)[M]_{i, j} & \text { if } i \neq j \\
\lambda_{i}[M]_{i, j} & \text { otherwise }
\end{array}\right.
$$

in which $M$ is a positive definite matrix of dimension $n . \lambda_{i}$ is the forgetting factor correspond with the $i$-th parameter. $i=1, \ldots, n$. It is worth to note that when the same $\lambda_{i}$ is applied to all parameters, the algorithm will be identical to the single forgetting factor scheme described in Section 3.1. In the proposed method, the cross terms of $F_{\lambda}(R)$ are weighted by the smallest forgetting factor and the diagonal terms are updated by the corresponding forgetting factors, hence, the multiple forgetting factors scheme is realized.

\subsection{The Inplementation of MFFRLS on Batteriey Parameter Identification}

In order to implement the proposed MFFRLS to the battery model. The characteristics of the ECM described in Equations (1) and (2) should be transformed to the form of Equation (6). Applying Laplace transform to the Equations (1) and (2), the battery dynamic can be expressed in the s-domain:

$$
\begin{gathered}
U_{p}(s)=\frac{R_{p}}{R_{p} C_{p} s+1} i(s) \\
U_{o c}(s)=U_{t}(s)+i(s) R_{0}+U_{p}(s)
\end{gathered}
$$

Considering the $i(s)$ and $\left(U_{t}-U_{o c}\right)(s)$ to be the system input and output respectively, the transfer function of the system can be obtained in Equation (14):

$$
\frac{\left(U_{t}-U_{o c}\right)(s)}{i(s)}=-R_{0}-\frac{R_{p}}{R_{p} C_{p} s+1}
$$

The forward Euler transformation method is utilized in this study to discretize the transfer function by substitute $(z-1) / T_{S}$ for $s$, in which $T_{S}$ is the sampling time:

$$
\begin{gathered}
\frac{\left(U_{t}-U_{o c}\right)(z)}{i(z)}=\frac{b_{0}+b_{1} z^{-1}}{1+a_{1} z^{-1}} \\
\left(U_{t}-U_{o c}\right)(k)=-a_{1}\left(U_{t}-U_{o c}\right)(k-1)+b_{0} i(k)+b_{1} i(k-1)
\end{gathered}
$$

in which

$$
\left\{\begin{array}{l}
a_{1}=\frac{T_{s}}{R_{p} C_{p}}-1 \\
b_{0}=-R_{0} \\
b_{1}=R_{0}-\frac{R_{o} T_{s}}{R_{p} C_{p}}-\frac{T_{s}}{C_{p}}
\end{array}\right.
$$


Equation (17) have the same form with Equation (6) and MFFRLS can then be realized to perform online identification of system parameters $a_{1}, b_{0}$ and $b_{1}$. The ECM parameters $R_{0}, R_{p}$ and $C_{p}$ can be calculated as follows:

$$
\left\{\begin{array}{l}
R_{0}=-b_{0} \\
R_{p}=\frac{a_{1} b_{0}-b_{1}}{a_{1}+1} \\
C_{p}=\frac{T_{s}}{a_{1} b_{0}-b_{1}}
\end{array}\right.
$$

\subsection{The Optimization of Forgeeting Factors}

In Section 3.3, the novel MFFRLS algorithm combining with the battery model is introduced. The model consists of three parameters and three different forgetting factors will be deployed. The problem is then converted to choosing the optimum value of these forgetting factors. The difficulty is that there are three different forgetting factors to be determined whose deviation from the optimum value will cause the underperformance of the system. To solve this multi-object optimization problem, an intelligent technique, known as particle swarm optimization (PSO), was utilized. PSO has been widely applied in optimization issues since originally proposed in 1995 for its simplicity and capability to achieve near optimal solution [47]. PSO is a swarm intelligence algorithm which consists a group of particles representing possible solutions. Each particle has two characteristics called position and velocity in order to simulate the movement of particles in the searching space. In each new iteration, the velocity of each particle is updated according to the personal best position and global best position with respect to the predefined fitness function $F_{T}$. The procedure of PSO is summarized in Table 2.

Table 2. Major steps of particle swarm optimization (PSO) optimization.

\begin{tabular}{|c|c|}
\hline Initialization & $\begin{array}{l}\text { Step 1: Define the number of particles } N \text {, maximum iteration } k_{\max } \text {, initial value and limitation } \\
\text { of velocity and position }\left(p_{j}^{0}, v_{j}^{0}, p_{\max }, p_{\min }, v_{\max }, v_{\min }\right) \text {, personal best position of each particle } \\
\text { pbest } t_{j} \text {, global best position of particles } g \text { best, inertia weight limitation }\left(w_{\max }, w_{\min }\right) \text { and } \\
\text { adjustment weight }\left(c_{1}, c_{2}\right) \text {. }\end{array}$ \\
\hline Updating & $\begin{array}{l}\text { Step 2: Update velocity and position of each particle using equations below }{ }^{1} \text {, if the calculated } \\
\text { value exceeds the limitation, then it will be replaced by the corresponding limit. } \\
w=w_{\max }-\left(w_{\max }-w_{\min }\right) k / k_{\max } \\
\left\{\begin{array}{l}v_{j}^{k+1}=w v_{j}^{k}+c_{1} r_{1}\left(\text { pbest }_{j}-x_{j}^{k}\right)+c_{2} r_{2}\left(\text { gbest }^{k}-x_{j}^{k}\right) \\
x_{j}^{k+1}=x_{j}^{k}+v_{j}^{k}\end{array}\right. \\
\text { Step 3: Calculate the fitness function of each particle, update the personal best position and } \\
\text { global best position by } \\
\left\{\begin{array}{l}\text { pbest } t_{j}=\min \left(F_{T j}, \text { pbest }\right. \\
\text { gbest }\end{array}\right) \min \left(F_{T j}, \text { gbest }\right)\end{array}$ \\
\hline Iteration and exit & Step 4: If $k=k_{\max }$, then exit and display the results, otherwise $k=k+1$ and return to step 2 \\
\hline
\end{tabular}

In this optimization process, the sum of the square error is defined as the fitness function expressed in Equation (19), as the identification accuracy is a fundamental indicator of the performance of the RLS algorithm.

$$
F_{T}=\sum_{k=1}^{L}\left[y(k)-\varphi^{T}(k) \hat{\theta}\right]^{2}
$$

In order to obtain the optimum forgetting factors of SFFRLS and MFFRLS, computer simulation of the PSO was conducted. The New European Driving Cycle (NEDC) experiment under the temperature of $25{ }^{\circ} \mathrm{C}$ was carried out and the recorded data was used to identify the parameters. $U_{o c}$ was derived from SOC-OCV curve characterized in Section 4.2 where SOC was accurately obtained from ampere-hour counting. The two PSO algorithms were initialized using the same parameter. The forgetting factors of SFFRLS and MFFRLS obtained through PSO optimization are listed in Table 3. 
Table 3. Forgetting factors optimized by PSO.

\begin{tabular}{cc}
\hline Algorithms & Forgetting Factors \\
\hline SFFRLS & 0.9689 \\
MFFRLS & {$[0.9272,0.9054,0.9062]$} \\
\hline
\end{tabular}

Note that the second forgetting factor and the third forgetting factor of the MFFRLS are numerically close. However, this is not by accident. According to Equation (17), system parameter $b_{0}=-R_{0}$ while $b_{1}=R_{0}-\left(R_{0} T_{s}\right) /\left(R_{p} C_{p}\right)-T_{s} / C_{p}$. The offline identification result in Section 4.2 shows that the value of $R_{0}$ and $R_{p}$ are as low as $10^{-2} \Omega$ order of magnitude while that of $C_{p}$ is $10^{3} F$ order of magnitude, $T_{s}=1 \mathrm{~s}$ in this study. Therefore, the last two terms of the expression of $b_{1}$ can be neglected and $b_{1} \approx R_{0}$. It can then be concluded that the second and third model parameters $b_{0}$ and $b_{1}$ have the same dynamic characteristic. As a consequence, the values of the optimum forgetting factors tend to be similar.

\subsection{Joint Algorithm of Online Parameter Identification and SOC Estimation}

The MFFRLS online identification method was combined with UKF SOC estimator to calculate the battery parameters and SOC precisely. The MFFRLS-UKF scheme is shown in Figure 2.

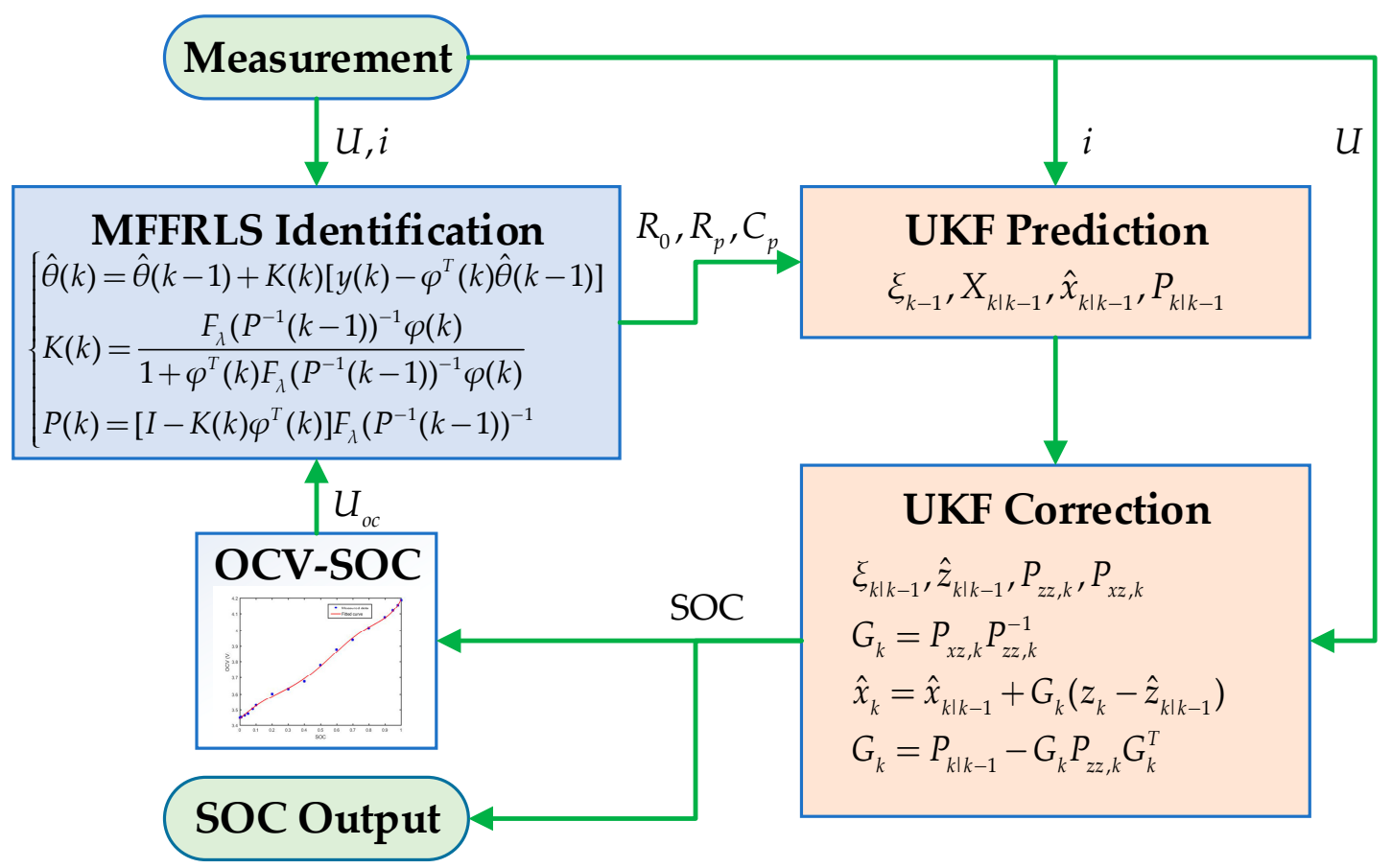

Figure 2. Diagram of the multiple forgetting factor recursive least square-unscented Kalman filter (MFFRLS-UKF) scheme.

As shown in Figure 2, the variables in MFFRLS and UKF are initialized. Each time the battery current and voltage are measured, using the measured current and voltage as well as the $U_{o c}$ derived from the SOC predicted by UKF, the MFFRLS can be conducted and battery parameters will be updated. The recently updated parameters will be transferred to the UKF section to replace the previous model parameters. The SOC is then estimated through UKF with the updated parameters, measured current and voltage. Then the algorithm moves to the next iteration. 


\section{Experimental Verifications}

\subsection{A Brief Introduction of the Test Bench}

The test of the battery is carried out on three major devices. The Arbin BT-5HC (Arbin Instruments, College Station, TX, USA) is used for battery charging, discharging and temperature monitoring. The Sanwood SC-80-CC-2 temperature chamber (Sanwood, Guangdong, China) provides a controlled environment of precise temperature and humidity for the battery. A host computer with Arbin Mits Pro Software (v7.0) and MATLAB R2016b (MathWorks, Natick, MA, USA) is used for charging and discharging control, data recording, and data processing. The commonly used cylindrical battery is chosen to carry out the experiment. The Samsung ICR18650-22P battery (Samsung SDI, Seoul, South Korea) is selected in this case whose technical specification includes: nominal capacity $2150 \mathrm{mAh}$, nominal voltage $3.6 \mathrm{~V}$, charging end voltage $4.2 \mathrm{~V}$, discharging cut-off voltage $2.75 \mathrm{~V}$. The overall scheme of the test system is illustrated in Figure 3.

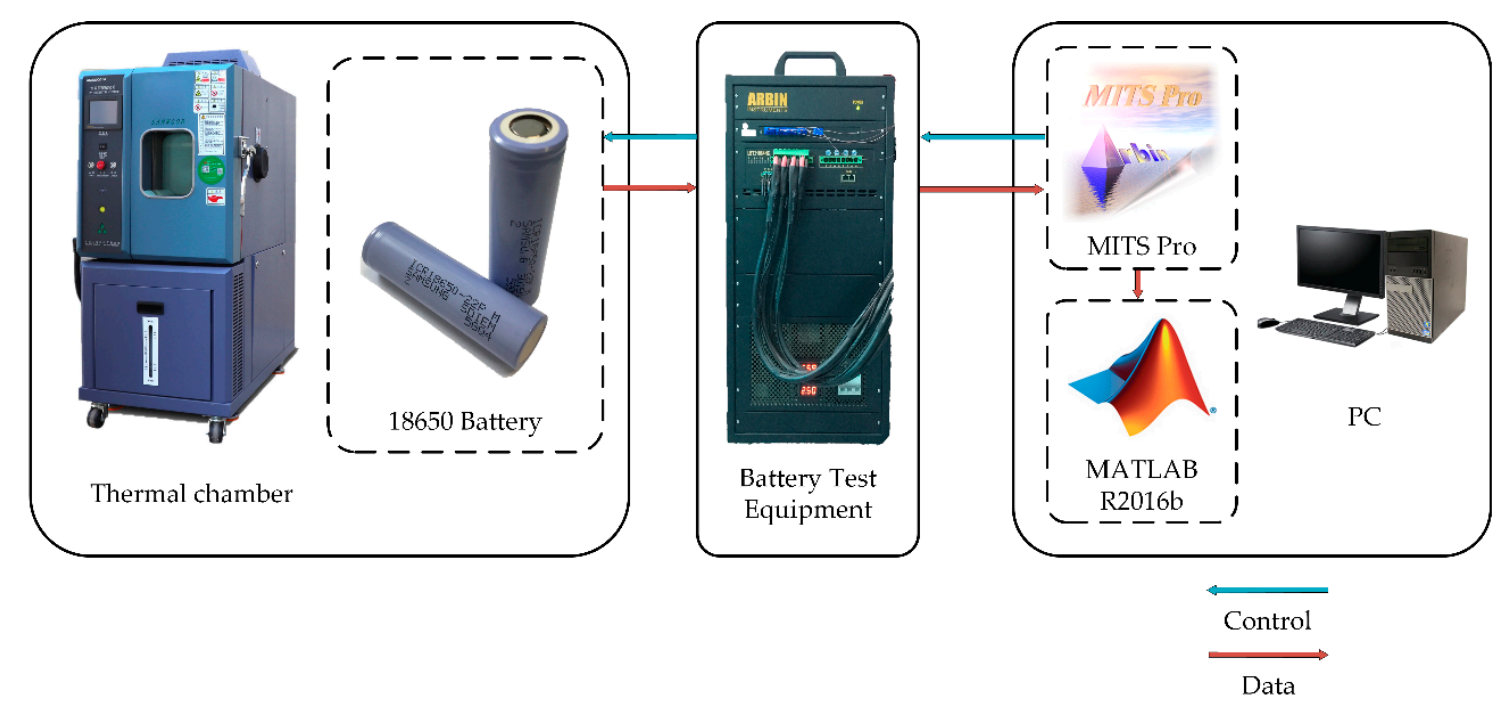

Figure 3. Outline of the test bench.

\subsection{Battery Characterization}

The basic characteristic of the battery should be calibrated, including the actual capacity, the SOC-OCV relationship and the offline identified parameters which are the model input of UKF algorithm and reference value of that identified by FFRLS-UKF and MFFRLS-UKF. The nominal capacity test was carried out at the temperature of $25^{\circ} \mathrm{C}$. The battery was fully charged through constant current constant voltage (CC-CV) method and then fully discharged to its cut-off voltage with $0.2 \mathrm{C}$ constant current, in which C-rate stands for the charge and discharge current relative to its nominal capacity, e.g., $1 \mathrm{C}$ is $2.15 \mathrm{~A}$ in this study. The procedure was repeated three times and the average amount of discharge was regarded as the actual capacity of the battery.

The SOC-OCV curve was derived through a series of pause discharge periods, the procedures are described as follows: (1) The battery was fully charged through CC-CV method and after 2 hours' rest, the measured terminal voltage was regarded as the OCV of the battery with $100 \%$ SOC; (2) the battery was discharged to $98 \%$ SOC with a constant current of $0.5 \mathrm{C}$ and then it was left in the open-circuit condition for $2 \mathrm{~h}$, the OCV correspond to $98 \%$ SOC can then be measured; (3) Step (2) was performed repeatedly to measure the OCV of the battery at SOC levels of $90 \%, 80 \%, 70 \%, 60 \%, 50 \%, 40 \%, 30 \%$, 
$20 \%, 10 \%, 8 \%, 5 \%, 3 \%, 1 \%$ and $0 \%$. The OCV under different SOC values was recorded and the 6-th order polynomial was utilized to describe the relationship between OCV and SOC:

$$
\mathrm{OCV}=\sum_{m=0}^{6} l_{m} \mathrm{SOC}^{m}
$$

The fitting result is listed in Table 4, the recorded data with fitted curve are shown in Figure 4.

Table 4. Polynomial coefficient of SOC-OCV curve

\begin{tabular}{cccccccc}
\hline Coefficients & $\boldsymbol{l}_{\mathbf{0}}$ & $\boldsymbol{l}_{\mathbf{1}}$ & $\boldsymbol{l}_{\mathbf{2}}$ & $\boldsymbol{l}_{\mathbf{3}}$ & $\boldsymbol{l}_{\mathbf{4}}$ & $\boldsymbol{l}_{\mathbf{5}}$ & $\boldsymbol{l}_{\mathbf{6}}$ \\
\hline Values & 3.4453 & 0.8606 & -0.0442 & -8.6603 & 30.2828 & -36.1564 & 14.4612 \\
\hline
\end{tabular}

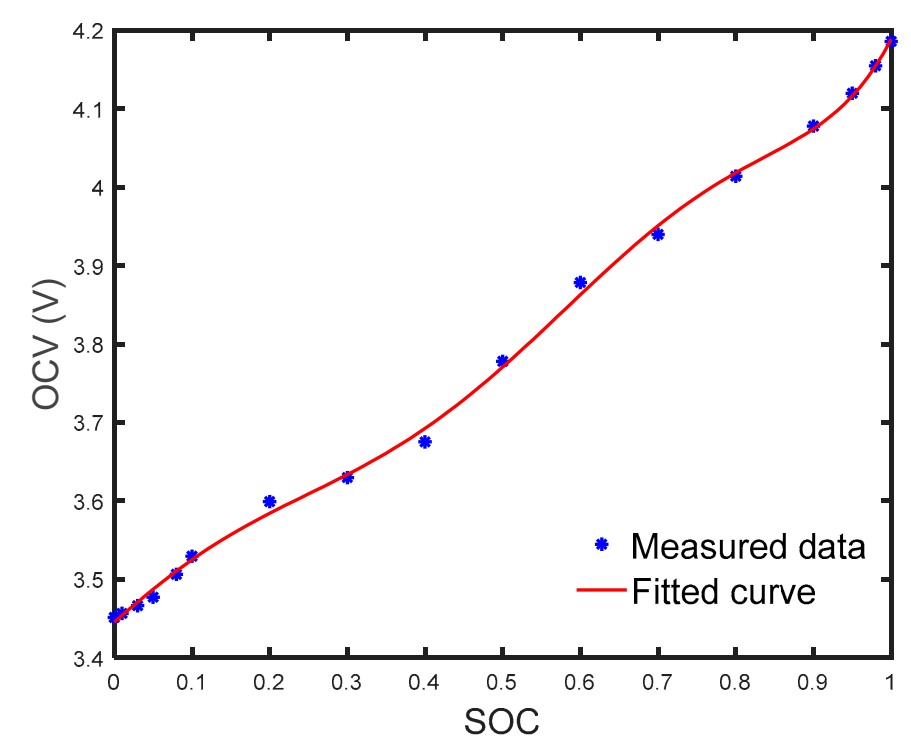

Figure 4. The measured data and fitted curve of OCV and SOC.

The reference values of the battery parameters were identified offline. The battery was discharged with a constant current and rested for $2 \mathrm{~h}$. The internal resistance was calculated from the instantaneous terminal voltage change $\Delta U_{t}$ after the disappearance of the constant discharge current $i$ as expressed in Equation (21). Then in the rest period, the variation of terminal voltage $U_{t}$ can be deduced as Equation (22) according to Equations (1) and (2), the polarization resistance and capacity were derived from the exponential curve fitting method. It is noteworthy to mention that the internal resistance identified offline is more reliable while other offline parameters may have considerable errors [48], nonetheless, the order of magnitude is a worthy point of reference as analyzed in Section 3.4. The values of the internal resistance obtained through offline identification method at 16 different SOC levels in the temperature of $25^{\circ} \mathrm{C}$ are listed in Table 5 . The values of all model parameters identified in $25^{\circ} \mathrm{C}$ at the SOC level of $98 \%$ are listed in Table 6 and utilized as the model parameters of UKF described in Sections 4.3-4.5.

$$
\begin{gathered}
R_{0}=\left|\frac{\Delta U_{t}}{i}\right| \\
U_{t}=U_{o c}-i R_{p} \exp \left(-\frac{t}{R_{p} C_{p}}\right)
\end{gathered}
$$


Table 5. Reference values of $R_{0}$ at different SOC levels in $25^{\circ} \mathrm{C}$.

\begin{tabular}{ccccccccc}
\hline SOC $(\%)$ & 98 & 95 & 90 & 80 & 70 & 60 & 50 & 40 \\
\hline$R_{0}(\Omega)$ & 0.0367 & 0.0369 & 0.0373 & 0.0368 & 0.0363 & 0.0359 & 0.0360 & 0.0364 \\
\hline SOC $(\%)$ & 30 & 20 & 10 & 8 & 5 & 3 & 1 & 0 \\
\hline$R_{0}(\Omega)$ & 0.0367 & 0.0374 & 0.0393 & 0.0410 & 0.0430 & 0.0456 & 0.0502 & 0.0522 \\
\hline
\end{tabular}

Table 6. Reference values of 1-RC model parameters in $25^{\circ} \mathrm{C}$ at $98 \%$ SOC level.

\begin{tabular}{cccc}
\hline Parameters & $\boldsymbol{R}_{0}(\boldsymbol{\Omega})$ & $\boldsymbol{R}_{\boldsymbol{p}}(\boldsymbol{\Omega})$ & $\boldsymbol{C}_{\boldsymbol{p}}(\mathbf{F})$ \\
\hline Values & 0.0367 & 0.0183 & 3768 \\
\hline
\end{tabular}

\subsection{NEDC Test under Constant Temperature}

The NEDC was loaded to the battery in a constant temperature of $25{ }^{\circ} \mathrm{C}$ to verify the effectiveness of three different methods under the ideal operation condition. Gaussian white noise sequences with variance $10^{-4}$ and $10^{-6}$ were added to measured current and voltage data respectively to simulate actual operation condition in all tests. The UKF algorithm using offline identified parameters as listed in Table 5 was employed as a contrast. Two online parameter identification methods combining UKF: the SFFRLS-UKF and the proposed MFFRLS-UKF, using the forgetting factors determined in Section 3.4, were implemented here and in the next three tests. The current variation of the NEDC cycle is shown in Figure 5.

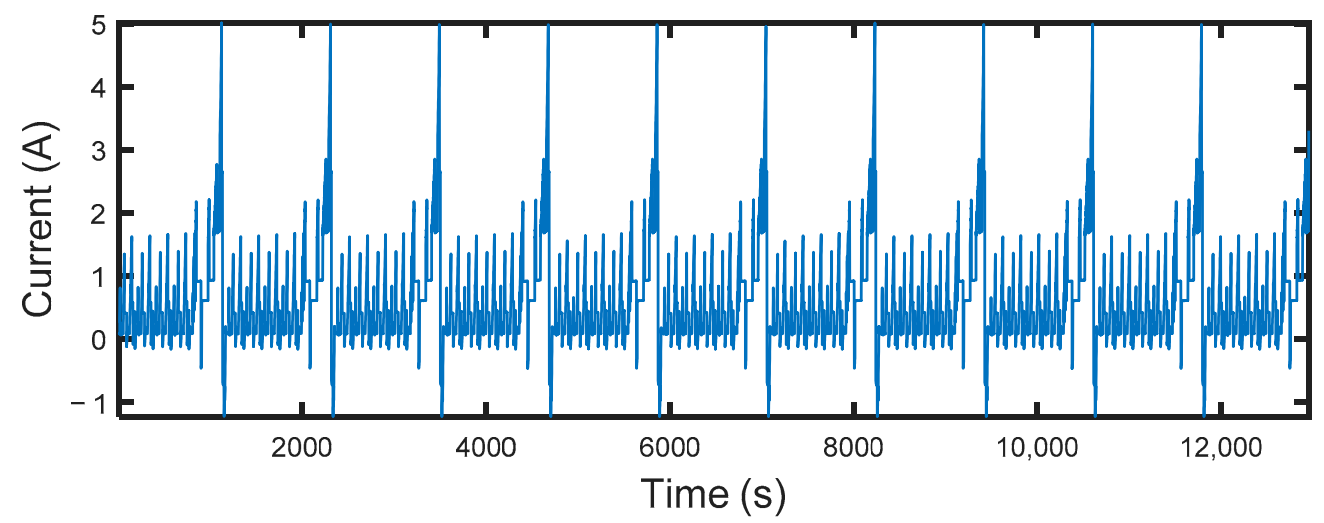

Figure 5. Current profile of New European Driving Cycle (NEDC) test.

The identification results of internal resistant $R_{0}$ of SFFRLS-UKF and MFFRLS-UKF are shown in Figure 6. Good caution must be taken into account that the $R_{0}$ changes steadily in a constant temperature environment, hence, a simple first-order lag filter is adopted here to smooth the identification result as expressed in Equation (23), in which $\eta$ is a constant between 0 and 1. Larger $\eta$ takes the advantage of higher sensitivity but decreases stability. Based on comparison experiments, $\eta$ is selected as 0.05 . The reference values are obtained through offline identification listed in Table 6 . It is clearly shown in Figure 6 that both the two algorithms provide reasonable results whose values are close to the reference value. The value of $R_{0}$ identified online rises quickly at the end of the test during which the SOC is low, which conforms with the offline identification results. 


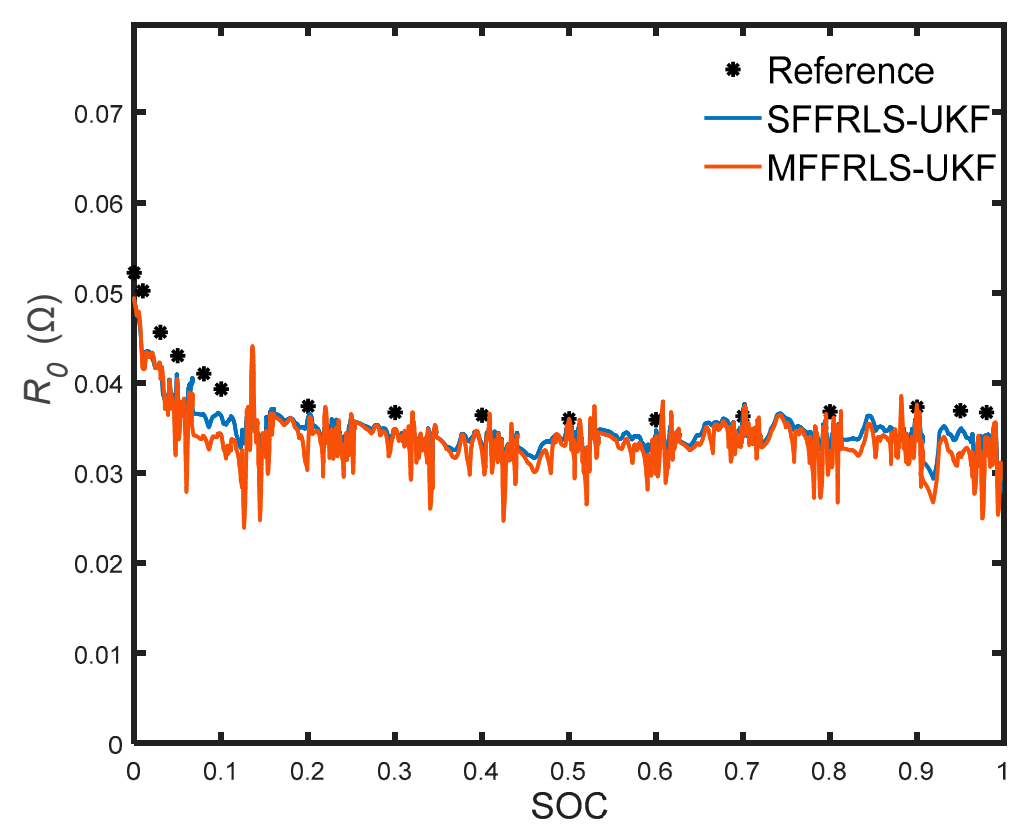

Figure 6. Identification result of $R_{0}$ by SFFRLS-UKF and MFFRLS-UKF.

$$
R_{0}^{\prime}(k)=(1-\eta) R_{0}^{\prime}(k-1)+\eta R_{0}(k)
$$

The result of SOC separately estimated by three different algorithms: UKF, SFFRLS-UKF and MFFRLS-UKF are listed in Table 7 and shown in Figure 7. SOC obtained by the ampere-hour integral method using a high-definition measurement of current and voltage was regarded as the reference SOC.

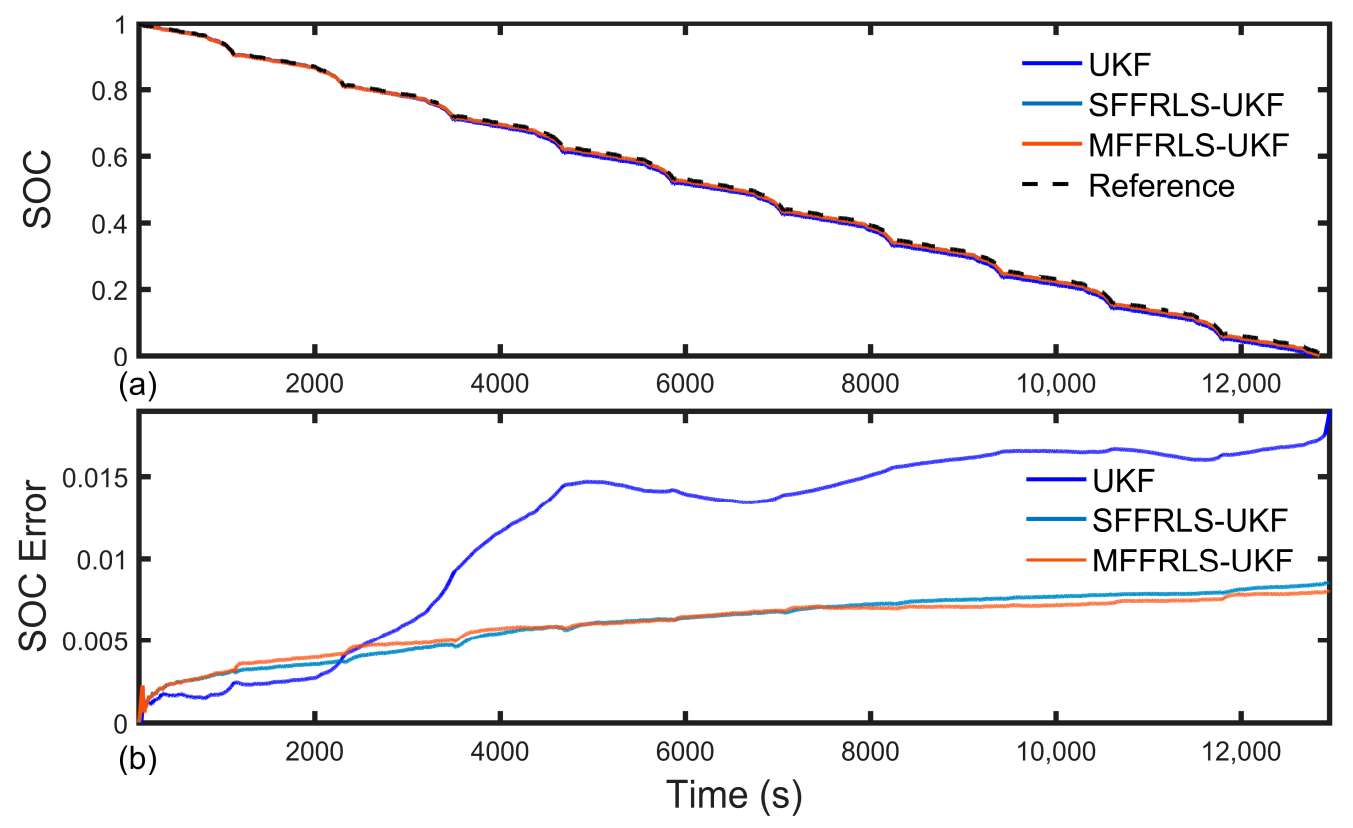

Figure 7. SOC estimation result of UKF, SFFRLS-UKF and MFFRLS-UKF during NEDC test under $25^{\circ} \mathrm{C}$ constant temperature: (a) SOC estimation; (b) SOC estimation error. 
Table 7. Statistic characters of SOC estimation of constant temperature NEDC test.

\begin{tabular}{cccc}
\hline Algorithms & UKF & SFFRLS-UKF & MFFRLS-UKF \\
\hline Mean error & 0.0120 & 0.0061 & 0.0060 \\
Max error & 0.0190 & 0.0085 & 0.0080 \\
RMSE & 0.0131 & 0.0063 & 0.0062 \\
\hline
\end{tabular}

Even though the UKF used the parameters identified in the same temperature, it can be seen in Table 7 that it shown the worst performance considering all statistic character values. This is due to the highly dynamic characters of the battery parameters, which are not only affected by the ambient temperature, but also influenced by other variables such as SOC and the aging of the battery. Thus, fixed parameters defined in a particular condition was not capable to catch up with the dynamic behavior of the battery, which resulted in a relatively poor estimation of SOC. The two online parameter identification methods shown batter results compare with the offline method, the mean errors of SOC of SFFRLS-UKF and MFFRLS-UKF were $0.61 \%$ and $0.60 \%$ respectively. Under the condition that the temperature remained constant, the parameters tended to change at a relatively slow rate, as a consequence, the two approaches had a similar performance and the advantage of the multiple forgetting factor scheme, specifically its fast tracking ability, was not yet clearly revealed.

\subsection{NEDC Test under Variable Temperature}

In order to verify the ability of capturing dynamic characteristic of batteries of three different methods and simulate the temperature changes during actual operation (e.g., cold start process in winter of high latitude areas) simultaneously, the NEDC cycles were loaded on the battery whose ambient temperature was changing during the whole test. The images of current and temperature as functions of time are shown in Figure 8.
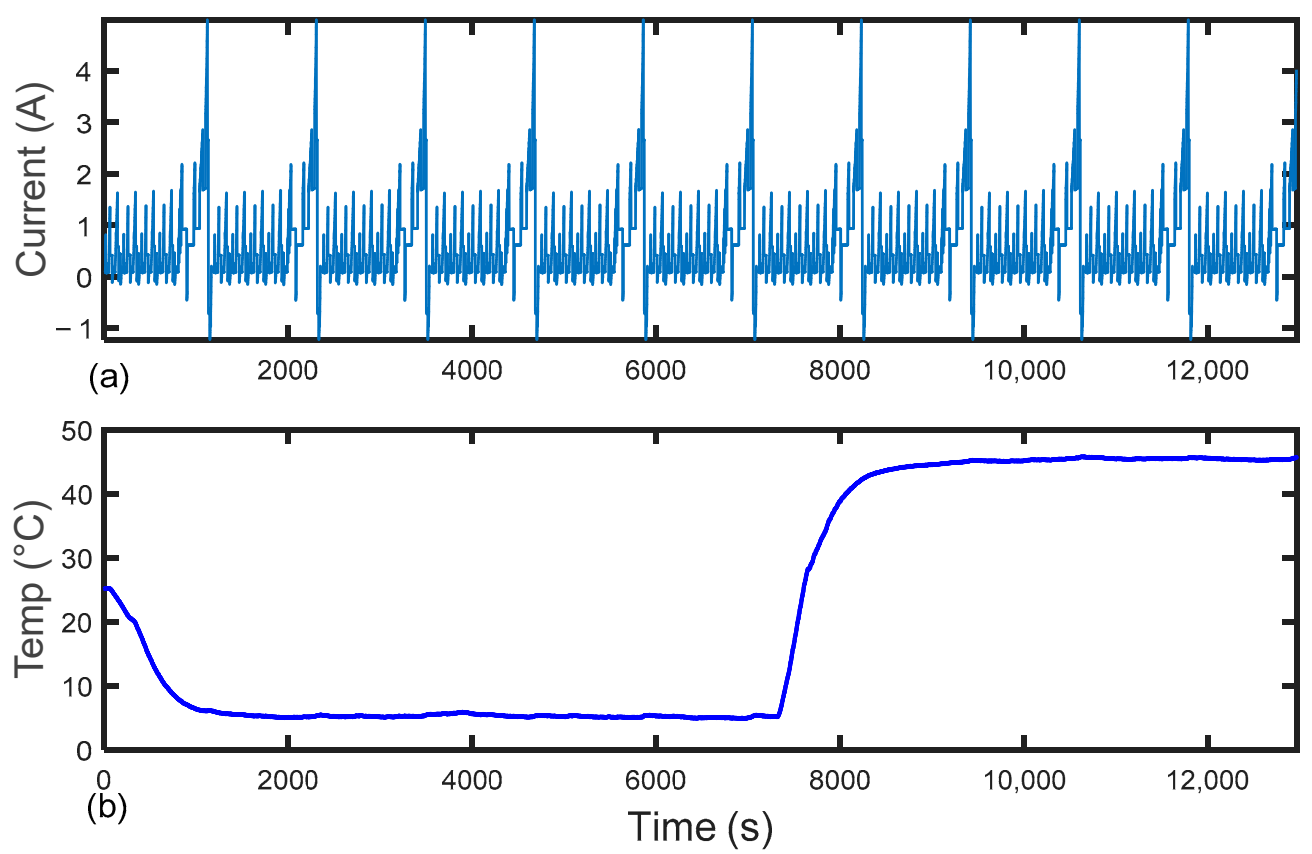

Figure 8. Current and temperature profile of NEDC test under variable temperature: (a) current; (b) temperature.

The SOC estimation results are shown in Table 8 and Figure 9. The two online identification algorithms, together with the UKF estimator, outperformed the single UKF estimator with offline battery parameters, indicating that the online identification methods could overcome the uncertainty of parameters caused by the variation of ambient temperature and SOC. The proposed MFFRLS-UKF 
method provided a precise SOC estimation with the $0.29 \%$ mean error of SOC, while the mean error of the traditional single forgetting factor method is $0.82 \%$. The novel MFFRLS-UKF displayed its superiority in the complex temperature changing environment.

Table 8. Statistic characters of SOC estimation of NEDC test under variable temperature.

\begin{tabular}{cccc}
\hline Algorithms & UKF & SFFRLS-UKF & MFFRLS-UKF \\
\hline Mean error & 0.0440 & 0.0082 & 0.0029 \\
Max error & 0.0600 & 0.0123 & 0.0047 \\
RMSE & 0.0477 & 0.0088 & 0.0030 \\
\hline
\end{tabular}
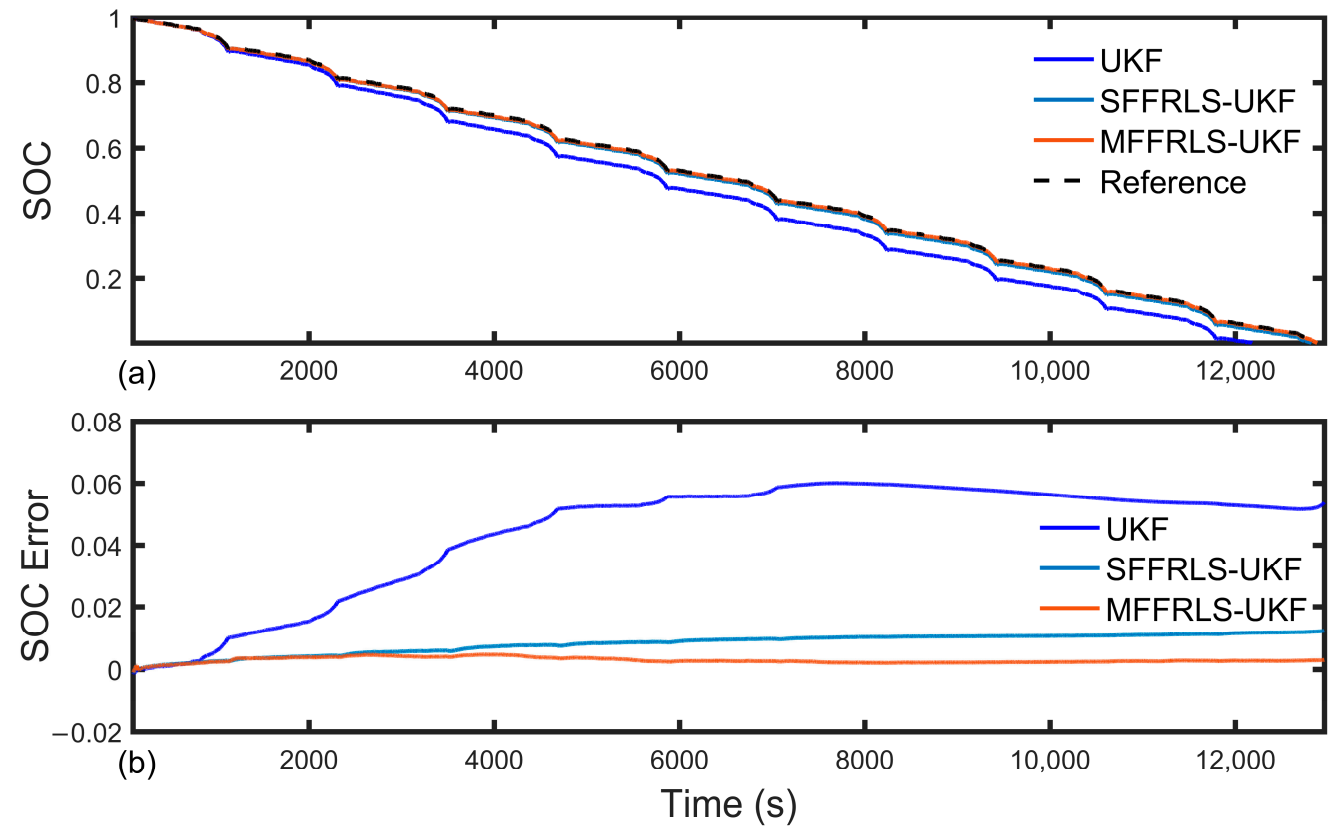

Figure 9. SOC estimation result of UKF, SFFRLS-UKF and MFFRLS-UKF during NEDC test under variable temperature: (a) SOC estimation; (b) SOC estimation error.

\subsection{Test of Multiple Cycles under Variable Temperature}

To further investigate the capability of tracking the changing battery characters of the proposed algorithm and its adaptability to different operation cycles. Two multiple cycles were deliberately designed and experiments were carried out on the battery under the changing ambient temperature. The two multiple cycles, consisting of Urban Dynamometer Driving Schedule (UDDS), Dynamic Street Test (DST), NEDC, and Federal Urban Driving Schedule (FUDS), named Multiple Cycle 1 and Multiple Cycle 2, respectively, were shown in Figure 10 in details about current and ambient temperature. Cycle 1 and Cycle 2 shared the same temperature trend but differed in the sequence of testing scheme, providing a comprehensive operation scene of the battery.

It can be seen in the SOC estimation result shown in Figure 11 and Table 9 that the proposed MFFRLS-UKF shows the highest precision in both the two multiple cycles. The mean error of SOC for SFFRLS-UKF and MFFRLS-UKF were $1.11 \%$ and $0.76 \%$ respectively in Multiple Cycle 1, 1.26\% and $0.85 \%$ respectively in Multiple Cycle 2, indicating that the proposed multiple forgetting factor identification scheme is good at handling the parameter uncertainty caused by the rapid changing of ambient environment and the vast variation in operation cycles. 

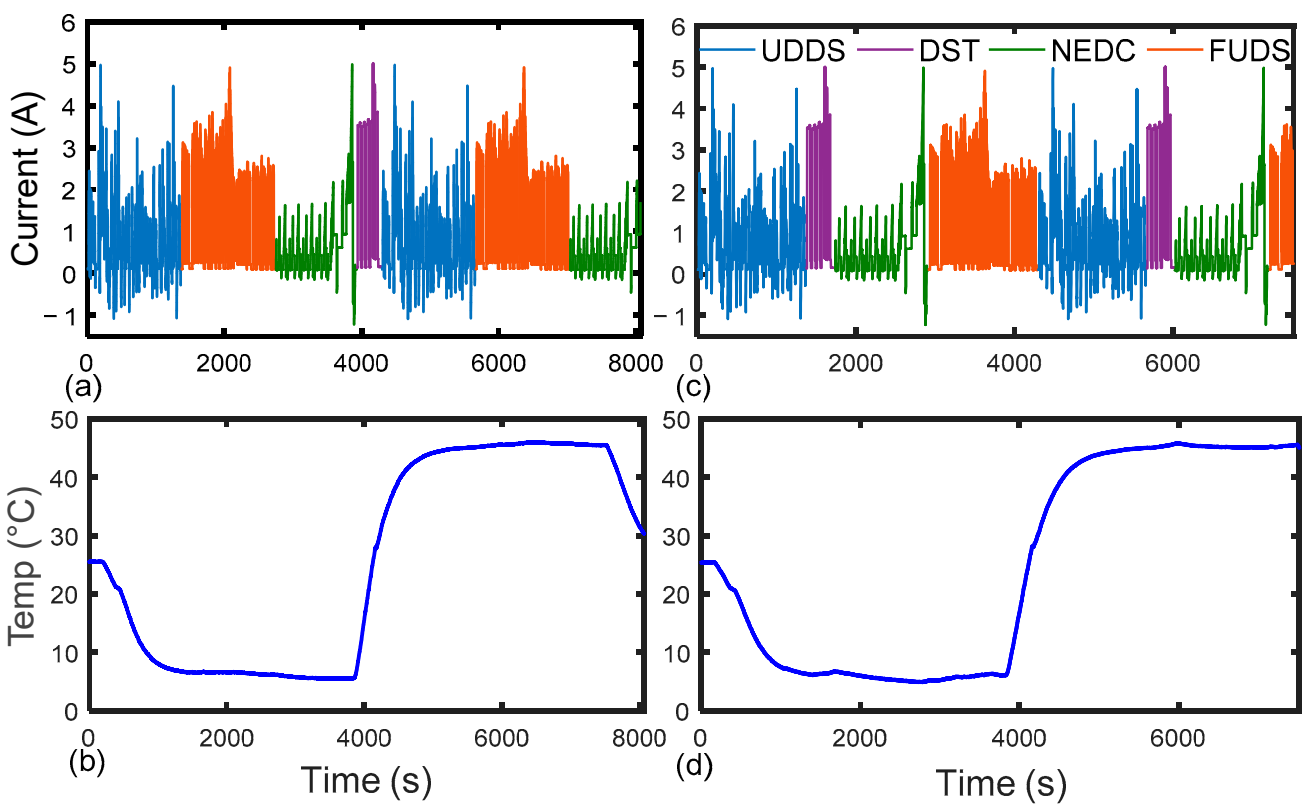

Figure 10. Current and temperature profile of two multiple cycles tests under changing temperature: (a,b) Multiple Cycle 1; (c,d) Multiple Cycle 2.
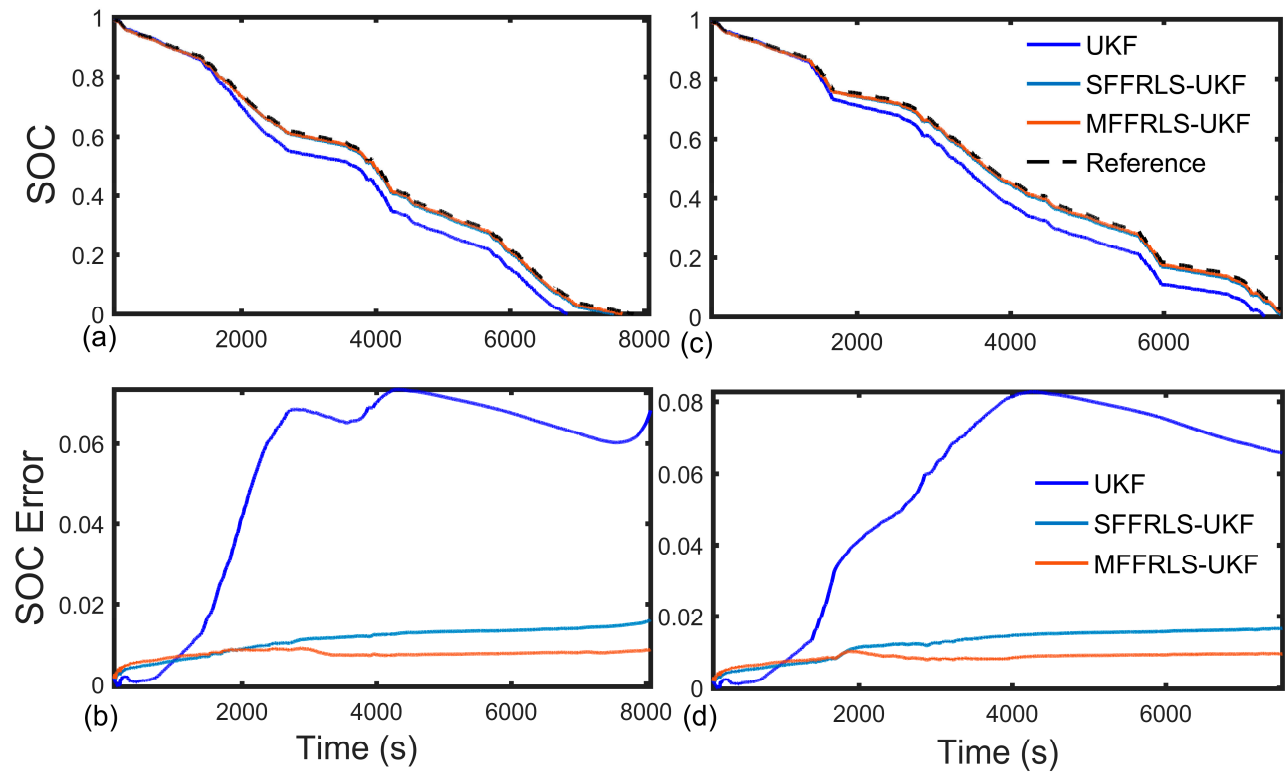

Figure 11. SOC estimation result and SOC error of UKF, SFFRLS-UKF and MFFRLS-UKF during multiple cycles tests under variable temperature: $(\mathbf{a}, \mathbf{b})$ Multiple Cycle 1; (c,d) Multiple Cycle 2.

Table 9. Statistic characters of SOC estimation under multiple cycles tests.

\begin{tabular}{ccccccc}
\hline Tests & \multicolumn{3}{c}{ Multiple Cycle 1 } & \multicolumn{3}{c}{ Multiple Cycle 2 } \\
\hline Algorithms & UKF & SFFRLS-UKF & MFFRLS-UKF & UKF & SFFRLS-UKF & MFFRLS-UKF \\
\hline Mean error & 0.0528 & 0.0111 & 0.0076 & 0.0558 & 0.0126 & 0.0085 \\
Max error & 0.0732 & 0.0160 & 0.0090 & 0.0827 & 0.0166 & 0.0103 \\
RMSE & 0.0583 & 0.0116 & 0.0076 & 0.0623 & 0.0132 & 0.0086 \\
\hline
\end{tabular}




\section{Conclusions}

In this paper, a novel MFFRLS procedure is introduced for the online identification of lithium-ion batteries to cope with the problems caused by different dynamics of battery parameters that cannot be easily solved by the SFFRLS method. Combined with UKF, the merit of estimating battery SOC with high accuracy of the proposed algorithm is verified through experiments under different temperature variations.

In the constant temperature NEDC test, it is found that the proposed MFFRLS-UKF has similar performance compared with SFFRLS-UKF in terms of SOC estimation accuracy. In the NEDC test and two multiple cycle tests under variable temperature, the MFFRLS-UKF outperforms SFFRLS-UKF and UKF with fixed parameters. (e.g., the mean SOC error of MFFRLS-UKF is reduced to $0.29 \%$ in NEDC test under changing temperature). Therefore, the proposed method is advantageous for SOC estimation, especially when faced with uncertainty of ambient temperature.

The online parameter identification combined with SOC estimation was performed in this study. The internal resistance identified by MFFRLS fluctuates more, as the Lithium-ion battery is a complex dynamic system, the model parameters identified by MFFRLS adjust quickly to adapt to the dynamic operation condition and provide an accurate SOC estimation. This fluctuation is preferable for SOC estimation but is not preferred for $R_{0}$-based $\mathrm{SOH}$ estimation. To address this trade-off issue, our future work will be focused on the joint estimation algorithm of SOC and $\mathrm{SOH}$ based on an intelligent online parameter identification method to achieve more comprehensive features of the BMS.

Author Contributions: B.X. and R.H. proposed the novel identification algorithm and analyzed the data; Z.L. performed the experiments; R.Z., Y.L. and W.Z. provided experimental guidance; H.W., W.W. and M.W. prepared necessary materials and equipment; R.H. wrote the paper.

Funding: This research was funded by the Shenzhen Economic, Trade and Information Commission of Shenzhen Municipality Strategic Emerging Industries and Future Industrial Development "Innovation Chain + Industrial Chain" Project (2017) and National Natural Science Foundation of China (Grant No. 51877120).

Conflicts of Interest: The authors declare no conflict of interest.

\section{Nomenclature}

\begin{tabular}{llll} 
Acronyms & & \multicolumn{2}{l}{ Symbols } \\
1-RC & First-order resistor-capacitor & $a, b$ & Parameters to be identified \\
2-RC & Second-order resistor-capacitor & $C_{n}$ & Nominal Capacity \\
ARX & Auto regressive exogenous & $C_{p}$ & Polarization capacity \\
BMS & Battery management system & $F_{\lambda}$ & Forgetting map \\
CC-CV & Constant current-constant voltage & $F_{T}$ & Fitness function \\
CKF & Cubature Kalman filter & $i$ & Load current \\
DST & Dynamic Street Test & $I$ & Identical matrix \\
ECM & Equivalent circuit model & $k$ & Time step index \\
EKF & Extended Kalman filter & $l$ & Coefficient of polynomial function \\
EV & Electric vehicle & $L$ & Number of observations \\
FUDS & Federal Urban Driving Schedule & $M$ & Positive definite matrix \\
LS & Least squares & $n$ & Dimension of parameters \\
MFF & Multiple forgetting factors & $n_{a}, n_{b}, d$ & SISO system constants \\
MFFRLS & Multiple forgetting factors recursive least square & $P$ & Covariance vector \\
NEDC & New European Driving Cycle & $R_{0}$ & Internal resistance \\
NEVs & New energy vehicles & $R_{p}$ & Polarization resistance \\
OCV & Open circuit voltage & $s$ & Complex variable \\
PC & Personal computer & $t$ & Time \\
PNGV & Partnership for a New Generation of Vehicles & $T_{S}$ & Sampling time \\
PSO & Particle swarm optimization & $U_{o c}$ & Open circuit voltage \\
RC & Resistor-capacitor & $U_{t}$ & Terminal voltage
\end{tabular}




\begin{tabular}{llll} 
RELS & Recursive extended least square & $V$ & Least square error function \\
RLS & Recursive least square & $y$ & Output of SISO system \\
RMSE & Root mean square error & $z$ & Discretization variable \\
RTLS & Recursive total least square & \multicolumn{1}{l}{ Greek symbols } \\
SISO & Single input single output & $\zeta$ & White noise \\
SOC & State of charge & $\eta$ & Filtering constant \\
SOH & State of health & $\theta$ & Parameter vector \\
SFFRLS & Single forgetting factor recursive least square & $\lambda$ & Forgetting factor \\
U-D & Unit upper triangular factor-diagonal factor & $\varphi$ & Data vector \\
UDDS & Urban Dynamometer Driving Schedule & & \\
UKF & Unscented Kalman filter & & \\
Subscript & & Superscript \\
min & Minimum value & $\prime$ & Filtered value \\
max & Maximum value & &
\end{tabular}

\section{References}

1. Zheng, Y.; Ouyang, M.; Han, X.; Lu, L.; Li, J. Investigating the error sources of the online state of charge estimation methods for lithium-ion batteries in electric vehicles. J. Power Sources 2018, 377, 161-188. [CrossRef]

2. Chen, X.; Shen, W.; Cao, Z.; Kapoor, A. A novel approach for state of charge estimation based on adaptive switching gain sliding mode observer in electric vehicles. J. Power Sources 2014, 246, 667-678. [CrossRef]

3. Zhang, R.; Xia, B.; Li, B.; Cao, L.; Lai, Y.; Zheng, W.; Wang, H.; Wang, W. State of the Art of Lithium-Ion Battery SOC Estimation for Electrical Vehicles. Energies 2018, 11, 1820. [CrossRef]

4. Lu, L.; Han, X.; Li, J.; Hua, J.; Ouyang, M. A review on the key issues for lithium-ion battery management in electric vehicles. J. Power Sources 2013, 226, 272-288. [CrossRef]

5. Panchal, S.; Mathew, M.; Fraser, R.; Fowler, M. Electrochemical thermal modeling and experimental measurements of 18650 cylindrical lithium-ion battery during discharge cycle for an EV. Appl. Therm. Eng. 2018, 135, 123-132. [CrossRef]

6. Tang, X.; Zou, C.; Yao, K.; Chen, G.; Liu, B.; He, Z.; Gao, F. A fast estimation algorithm for lithium-ion battery state of health. J. Power Sources 2018, 396, 453-458. [CrossRef]

7. Xia, B.; Chen, C.; Tian, Y.; Sun, W.; Xu, Z.; Zheng, W. A novel method for state of charge estimation of lithium-ion batteries using a nonlinear observer. J. Power Sources 2014, 270, 359-366. [CrossRef]

8. Ng, K.S.; Moo, C.-S.; Chen, Y.-P.; Hsieh, Y.-C. Enhanced coulomb counting method for estimating state-of-charge and state-of-health of lithium-ion batteries. Appl. Energy 2009, 86, 1506-1511. [CrossRef]

9. Tong, S.; Klein, M.P.; Park, J.W. On-line optimization of battery open circuit voltage for improved state-of-charge and state-of-health estimation. J. Power Sources 2015, 293, 416-428. [CrossRef]

10. Xiong, R.; He, H.; Sun, F.; Zhao, K. Evaluation on State of Charge Estimation of Batteries With Adaptive Extended Kalman Filter by Experiment Approach. IEEE Trans. Veh. Technol. 2013, 62, 108-117. [CrossRef]

11. Plett, G.L. Extended Kalman filtering for battery management systems of LiPB-based HEV battery packs. J. Power Sources 2004, 134, 277-292. [CrossRef]

12. Tian, Y.; Xia, B.; Sun, W.; Xu, Z.; Zheng, W. A modified model based state of charge estimation of power lithium-ion batteries using unscented Kalman filter. J. Power Sources 2014, 270, 619-626. [CrossRef]

13. He, W.; Williard, N.; Chen, C.; Pecht, M. State of charge estimation for electric vehicle batteries using unscented kalman filtering. Microelectron. Reliab. 2013, 53, 840-847. [CrossRef]

14. Xia, B.; Wang, H.; Tian, Y.; Wang, M.; Sun, W.; Xu, Z. State of Charge Estimation of Lithium-Ion Batteries Using an Adaptive Cubature Kalman Filter. Energies 2015, 8, 5916-5936. [CrossRef]

15. Xia, B.; Wang, H.; Wang, M.; Sun, W.; Xu, Z.; Lai, Y. A New Method for State of Charge Estimation of Lithium-Ion Battery Based on Strong Tracking Cubature Kalman Filter. Energies 2015, 8, 13458-13472. [CrossRef]

16. Xia, B.; Sun, Z.; Zhang, R.; Lao, Z. A Cubature Particle Filter Algorithm to Estimate the State of the Charge of Lithium-Ion Batteries Based on a Second-Order Equivalent Circuit Model. Energies 2017, 10, 457. [CrossRef] 
17. Kim, I.-S. The novel state of charge estimation method for lithium battery using sliding mode observer. J. Power Sources 2006, 163, 584-590. [CrossRef]

18. Tian, Y.; Chen, C.; Xia, B.; Sun, W.; Xu, Z.; Zheng, W. An Adaptive Gain Nonlinear Observer for State of Charge Estimation of Lithium-Ion Batteries in Electric Vehicles. Energies 2014, 7, 5995-6012. [CrossRef]

19. Lin, C.; Mu, H.; Xiong, R.; Shen, W. A novel multi-model probability battery state of charge estimation approach for electric vehicles using H-infinity algorithm. Appl. Energy 2016, 166, 76-83. [CrossRef]

20. Zhu, Q.; Li, L.; Hu, X.; Xiong, N.; Hu, G.-D. Ho-Based Nonlinear Observer Design for State of Charge Estimation of Lithium-Ion Battery With Polynomial Parameters. IEEE Trans. Veh. Technol. 2017, 66, 10853-10865. [CrossRef]

21. Marongiu, A.; Nußbaum, F.G.W.; Waag, W.; Garmendia, M.; Sauer, D.U. Comprehensive study of the influence of aging on the hysteresis behavior of a lithium iron phosphate cathode-based lithium ion battery-An experimental investigation of the hysteresis. Appl. Energy 2016, 171, 629-645. [CrossRef]

22. Zhang, R.; Xia, B.; Li, B.; Lai, Y.; Zheng, W.; Wang, H.; Wang, W.; Wang, M. Study on the Characteristics of a High Capacity Nickel Manganese Cobalt Oxide (NMC) Lithium-Ion Battery-An Experimental Investigation. Energies 2018, 11, 2275. [CrossRef]

23. Yu, Q.; Xiong, R.; Lin, C.; Shen, W.; Deng, J. Lithium-Ion Battery Parameters and State-of-Charge Joint Estimation Based on H-Infinity and Unscented Kalman Filters. IEEE Trans. Veh. Technol. 2017, 66, 8693-8701. [CrossRef]

24. Tang, X.; Wang, Y.; Zou, C.; Yao, K.; Xia, Y.; Gao, F. A novel framework for Lithium-ion battery modeling considering uncertainties of temperature and aging. Energy Convers. Manag. 2019, 180, 162-170. [CrossRef]

25. He, Z.; Gao, M.; Wang, C.; Wang, L.; Liu, Y. Adaptive State of Charge Estimation for Li-Ion Batteries Based on an Unscented Kalman Filter with an Enhanced Battery Model. Energies 2013, 6, 4134-4151. [CrossRef]

26. Xiong, R.; Sun, F.; Chen, Z.; He, H. A data-driven multi-scale extended Kalman filtering based parameter and state estimation approach of lithium-ion olymer battery in electric vehicles. Appl. Energy 2014, 113, 463-476. [CrossRef]

27. Giordano, G.; Klass, V.; Behm, M.; Lindbergh, G.; Sjoberg, J. Model-Based Lithium-Ion Battery Resistance Estimation from Electric Vehicle Operating Data. IEEE Trans. Veh. Technol. 2018, 67, 3720-3728. [CrossRef]

28. Zhang, J.; Wei, Y.; Qi, H. State of charge estimation of $\mathrm{LiFePO}_{4}$ batteries based on online parameter identification. Appl. Math. Model. 2016, 40, 6040-6050. [CrossRef]

29. Wei, Z.; Zou, C.; Leng, F.; Soong, B.H.; Tseng, K.-J. Online Model Identification and State-of-Charge Estimate for Lithium-Ion Battery With a Recursive Total Least Squares-Based Observer. IEEE Trans. Ind. Electron. 2018, 65, 1336-1346. [CrossRef]

30. Wei, Z.; Meng, S.; Xiong, B.; Ji, D.; Tseng, K.J. Enhanced online model identification and state of charge estimation for lithium-ion battery with a FBCRLS based observer. Appl. Energy 2016, 181, 332-341. [CrossRef]

31. Rahimi-Eichi, H.; Baronti, F.; Chow, M.Y. Modeling and Online Parameter Identification of Li-Polymer Battery Cells for SOC estimation. In Proceedings of the 2012 IEEE International Symposium on Industrial Electronics, Hangzhou, China, 28-31 May 2012; pp. 1336-1341.

32. Feng, T.; Yang, L.; Zhao, X.; Zhang, H.; Qiang, J. Online identification of lithium-ion battery parameters based on an improved equivalent-circuit model and its implementation on battery state-of-power prediction. J. Power Sources 2015, 281, 192-203. [CrossRef]

33. Dai, H.; Xu, T.; Zhu, L.; Wei, X.; Sun, Z. Adaptive model parameter identification for large capacity Li-ion batteries on separated time scales. Appl. Energy 2016, 184, 119-131. [CrossRef]

34. Schwunk, S.; Straub, S.; Jung, M. Online Parameterization of a Function Describing the Open-Circuit Voltage by a Least Square Method with Adaptive Forgetting Factor. J. Electrochem. Soc. 2013, 160, A2155-A2159. [CrossRef]

35. Xiong, R.; He, H.; Sun, F.; Zhao, K. Online Estimation of Peak Power Capability of Li-Ion Batteries in Electric Vehicles by a Hardware-in-Loop Approach. Energies 2012, 5, 1455-1469. [CrossRef]

36. Verbrugge, M. Adaptive, multi-parameter battery state estimator with optimized time-weighting factors. J. Appl. Electrochem. 2007, 37, 605-616. [CrossRef]

37. Duong, V.-H.; Bastawrous, H.A.; Lim, K.; See, K.W.; Zhang, P.; Dou, S.X. Online state of charge and model parameters estimation of the $\mathrm{LiFePO}_{4}$ battery in electric vehicles using multiple adaptive forgetting factors recursive least-squares. J. Power Sources 2015, 296, 215-224. [CrossRef] 
38. Rijanto, E.; Rozaqi, L.; Nugroho, A.; Kanarachos, S. RLS with Optimum Multiple Adaptive Forgetting Factors for SoC and SoH Estimation of Li-Ion Battery. In Proceedings of the 2017 5th International Conference on Instrumentation, Control, and Automation (ICA), Yogyakarta, Indonesia, 9-11 August 2017; pp. 73-77.

39. Vahidi, A.; Stefanopoulou, A.; Peng, H. Recursive least squares with forgetting for online estimation of vehicle mass and road grade: Theory and experiments. Veh. Syst. Dyn. 2005, 43, 31-55. [CrossRef]

40. Uosaki, K.; Yotsuya, M.; Hatanaka, T. Adaptive identification of non-stationary systems with multiple forgetting factors. In Proceedings of the 35th IEEE Conference on Decision and Control, Kobe, Japan, 11-13 December 1996; pp. 851-856.

41. Hardier, G. An Extended U-D Algorithm with Multiple Forgetting Factors for RLS Estimation of Model Parameters. IFAC-PapersOnLine 2015, 48, 200-207. [CrossRef]

42. Saelid, S.; Foss, B. Adaptive controllers with a vector variable forgetting factor. In Proceedings of the $22 \mathrm{nd}$ IEEE Conference on Decision and Control, San Antonio, TX, USA, 14-16 December 1983; pp. 1488-1494.

43. Li, J.; Wang, L.; Lyu, C.; Liu, E.; Xing, Y.; Pecht, M. A parameter estimation method for a simplified electrochemical model for Li-ion batteries. Electrochim. Acta 2018, 275, 50-58. [CrossRef]

44. Panchal, S.; McGrory, J.; Kong, J.; Fraser, R.; Fowler, M.; Dincer, I.; Agelin-Chaab, M. Cycling degradation testing and analysis of a LiFePO4 battery at actual conditions. Int. J. Energy Res. 2017, 41, 2565-2575. [CrossRef]

45. Hu, X.; Li, S.; Peng, H. A comparative study of equivalent circuit models for Li-ion batteries. J. Power Sources 2012, 198, 359-367. [CrossRef]

46. Fraccaroli, F.; Peruffo, A.; Zorzi, M. A New Recursive Least Squares Method with Multiple Forgetting Schemes. In Proceedings of the IEEE Conference on Decision and Control (CDC), Osaka, Japan, 15-18 December 2015; pp. 3367-3372.

47. Tian, Y.; Li, D.; Tian, J.; Xia, B. State of charge estimation of lithium-ion batteries using an optimal adaptive gain nonlinear observer. Electrochim. Acta 2017, 225, 225-234. [CrossRef]

48. Lao, Z.; Xia, B.; Wang, W.; Sun, W.; Lai, Y.; Wang, M. A Novel Method for Lithium-Ion Battery Online Parameter Identification Based on Variable Forgetting Factor Recursive Least Squares. Energies 2018, 11, 1358. [CrossRef] 